\title{
ESTUDIO CUANTITATIVO DE LA ECOCARDIOGRAFÍA EQUINA
}

\section{QUANTITATIVE STUDY OF EQUINE ECHOCARDIOGRAPHY}

\author{
Carlos H. Lightowler ${ }^{1}$; Gabriela Pidal ${ }^{2}$; María Laura Cattáneo ${ }^{3}$
}

\begin{abstract}
SUMMARY
The authors carried out a study on differents echocardiographic values of the horse. They determined the following parameters: Left Ventricular Diastolic Diameter (LVDD), Left Ventricular Systolic Diameter (LVSD), Diastolic Thickness of Interventricular Septum (DTIS), Systolic Thickness of Interventricular Septum (STIS), Left Ventricular Diastolic Thickness of Free Wall (LVDTFW), Left Ventricular Systolic Thickness of Free Wall (LVSTFW), Diastolic Diameter of Left Atrium (DDLA), Diastolic Diameter of Aortic Ring (DDAR), Diastolic Diameter of Valsalva Sinus (DDVS), Diastolic Diameter of Aortic Root (DDAR), Diastolic Area of Aortic Ring (DAAR), Diastolic Perimeter of Aortic Ring (DPAR), Left Ventricular Diastolic Volume (LVDV), Left Ventricular Systolic Volume (LVSV), Left Ventricular Ejection Fraction (LVEF), Left Ventricular Fractional Shortening (LVFS), Velocity of Circumferencial Shortening (VCS), Left Ventricular Free Wall Fractional Thickening (LVFWFS), Fractional Thickening of Interventricular Septum (FTIS), E-point Septal Separation (EPSS), Normalized E-point Septal Separation (EPSSn), Left Ventricular Pre-ejection Period (LVPEP), Left Ventricular Ejection Time (LVET), Total electromechanical Systole (TES) and the LVPEP/LVET ratio.

Statistically we obtained the mean, standard deviation, coefficient of variation, median, the maximum and minimum values, first and third cuartil. Also the correlation between the EPSS and LVFS; EPSSn and LVFS; systolic intervals and heart rate; systolic intervals and body weight; ventricular diameters and body weight, ventricular volumes and body weight; parietal and septal thickness and body weight was also studied. Also, in the cases in which the correlation was significant the lineal regression was studied.

The obtained values of each one of the parameters studied are in the Tables I and II of the body of the article.
\end{abstract}

KEY wORDS: Echocardiography, Equine, Echocardiographic values.

Palabras Claves: Ecocardiografía, Equino, Valores ecocardiográficos.

\section{INTRODUCCIÓN}

Desde su introducción en la clínica equina [43, $44,45]$ la ecocardiografía ha incrementado su participación como herramienta diagnóstica y pronóstica en la evaluación de las enfermedades congénitas y adquiridas del corazón equino $[6,7$, $8,20,24]$.

1-2 Departamento de Medicina.

3 Departamento de Medicina Preventiva y Salud Pública. Facultad de Ciencias Veterinarias. Universidad de Buenos Aires. Avda. Chorroarin 280 (C1427 CWO) Buenos Aires, Argentina.
Asimismo, desempeña un importante papel en el estudio de las modificaciones que sufre el corazón como consecuencia de los distintos regímenes de entrenamiento empleados para las competencias en que intervienen equinos $[5,41,54]$.

Este procedimiento no sólo posee valor cualitativo (evaluación subjetiva de la contractilidad, estudio de la anatomía cardíaca) sino fundamentalmente cualitativo, a través de la medición de estructuras, determinación del volumen de cámaras y cálculo de índices que permiten evaluar la performance ventricular (intervalos sistólicos e índices de la fase de eyección) $[10,11,37,39]$.

Desde el punto de vista cuantitativo no son abundantes las publicaciones que ofrecen valores norma- 
les de los distintos parámetros ecocardiográficos de interés diagnóstico y muchos de ellos no son comparables entre sí, debido a la variable metodología utilizada para la recolección de los guarismos publicados [22, 23, 47, 50, 63].

El objetivo del presente estudio fue la obtención de valores normales para distintas estructuras anatómicas (tabique interventricular y pared libre del ventrículo izquierdo, diámetros, área y perímetro aórtico), volúmenes ventriculares, de los intervalos sistólicos y de los índices de la fase de eyección del ventrículo izquierdo en una población equina homogénea.

\section{MATERIALES Y MÉTODOS}

Para el desarrollo del presente protocolo se estudiaron 102 caballos carentes de evidencias clínicas de enfermedad cardíaca, los cuales fueron manejados sin ninguna forma de sedación. El grupo fue conformado por caballos mestizos, con edades comprendidas entre 3 y 14 años y pesos entre 296 y $546 \mathrm{~kg}$. Fue condición fundamental para la inclusión en el estudio que los animales no presentaran ninguna forma especial de entrenamiento.

Los estudios se realizaron colocando los animales en una manga, sin ninguna otra forma de sujeción física. Las imágenes se obtuvieron con ecógrafos marca KONTRON, modelos SIGMA 1 AC y SIGMA IRIS 440 , configurados con transductores sectoriales mecánicos de $2,9 \mathrm{MHz}$ (Tipo D) y 3,5 MHz. (Tipo C) con representación de la imagen en pantalla hasta una profundidad máxima de $23 \mathrm{~cm}$ y módulo de electrocardiografía. Como equipos periféricos se emplearon una videoimpresora marca SONY, modelo UP-870MD, y una videograbadora VHS marca Philips, modelo VR354.

Con el objeto de obtener registros de calidad comparable en todos los casos, antes de cada estudio se procedió a estandarizar el procesamiento de la imagen. Se utilizó el programa Cardio general de la configuración del ecógrafo. El resto de los controles se ajustó de la siguiente manera para ambos transductores: TGC máxima a todos los niveles de la imagen; Ganancia general para modo-B, $50 \%$ y para modo-M, 60\%; nivel de rechazo, $50 \%$; realce, 0 ; postprocesado 3; profundidad de foco, 5 con enfoque del transductor para campo lejano. No se empleó filtro de movimiento. Los electrodos para el control electrocardiográfico se colocaron de la siguiente manera: tierra de paciente (electrodo negro) sobre la gotera yugular derecha; electrodo rojo en la punta del cartílago cariniforme del esternón y el verde inmediatamente por delante del cartílago xifoides del esternón, sobre la línea media ventral, logrando así complejos ventriculares positivos.

Durante la realización de las ecocardiografías y con el objeto de mejorar la definición de las interfaces a identificar para la realización de las mediciones, sólo se modificó el nivel de la ganancia general y de rechazo de ecos, según el transductor empleado. Como técnica general del examen se empleó la metodología previamente descrita $[24,25,26,31]$.

Se determinaron los siguientes parámetros:

1. Medidas anatómicas: Diámetro Diastólico del Ventrículo Izquierdo (DDVI), Diámetro Sistólico del Ventrículo Izquierdo (DSVI), Espesor Diastólico del Tabique Interventricular (EDTI), Espesor Sistólico del Tabique Interventricular (ESTI), Espesor Diastólico de la Pared Posterior del ventrículo izquierdo (EDPP), Espesor Sistólico de la Pared Posterior del ventrículo izquierdo (ESPP), Diámetro Diastólico del Atrio Izquierdo (DDAI), Diámetro diastólico del Anillo Aórtico (DDAAo), Diámetro Diastólico del Seno de Valsalva (DDSV), Diámetro Diastólico de la Raíz Aórtica (DDRAo), Área Diastólica del Anillo Aórtico (ADAAo), Perímetro Diastólico del Anillo Aórtico (PDAAo), Volúmenes: Volumen Diastólico del Ventrículo Izquierdo (VDVI) y Volumen Sistólico del Ventrículo Izquierdo (VSVI).

2. Índices de la fase de eyección: Fracción de Acortamiento (FA), Fracción de Eyección (FE), Velocidad de Acortamiento Circunferencial (VAC), Engrosamiento Fraccional de la Pared Posterior del ventrículo izquierdo (EFPP), Engrosamiento Fraccional del Tabique Interventricular (EFTI), Separación Septal del Punto E (SSPE) y Separación Septal del Punto E normalizada (SSPEn).

3. Intervalos sistólicos: Período pre-eyectivo del ventrículo izquierdo (PPEVI), Tiempo de eyección del ventrículo izquierdo (TEVI), Sístole electromecánica total del ventrículo izquierdo (SETVI) y relación PPEVI/TEVI.

Las mediciones se realizaron a partir de imágenes en modo-M guiado, derivadas de ecotomogramas en modo-B registrados desde la ventana paraesternal derecha, salvo las correspondientes a la aorta y su válvula que se midieron en modo-B. El diámetro diastólico del atrio izquierdo se midió también en modo-B a partir de ecotomogramas en eje largo del tracto de entrada del ventrículo izquierdo obtenidos desde la ventana paraesternal izquierda. 
En todos los casos para efectuar las mediciones se siguieron lineamientos establecidos [31] y lo especificado por la American Society of Echocardiography, empleando los calibres del equipo $[19,38,51,52]$.

La medición de los distintos accidentes se realizó de la siguiente manera:

1. Diámetros ventriculares: a partir de ecotomogramas registrados en eje corto, a nivel de las cuerdas tendinosas, colocando el cursor de corte en un punto equidistante de ambos paquetes de cuerdas tendinosas. El diámetro diastólico se midió en telediástole (coincidentemente con el comienzo del complejo ventricular del electrocardiograma) y el diámetro sistólico en el punto de máxima excursión posterior del tabique interventricular.

2. Espesores: a partir de ecotomogramas registrados en eje corto, a nivel de las cuerdas tendinosas, colocando el cursor de corte en un punto equidistante de ambos paquetes de cuerdas tendinosas. El espesor diastólico del tabique interventricular se midió en telediástole (en correspondencia con el primer accidente del complejo ventricular del electrocardiograma) mientras que su espesor sistólico se midió en el punto de máxima excursión posterior. El espesor diastólico de la pared posterior del ventrículo izquierdo se midió en el mismo nivel que el espesor diastólico septal (telediástole), mientras que el espesor sistólico se determinó a nivel de su punto de máxima excursión anterior.

3. Aorta y su anillo: las mediciones de la aorta y su anillo valvular se realizaron todas en modo-B. El área y el perímetro del anillo aórtico se midió desde un ecotomograma en eje corto a nivel de la base cardíaca y la evaluación de los diámetros del anillo aórtico, del seno de Valsalva y raíz de la aorta se realizaron desde un ecotomograma en eje largo del tracto de salida del ventrículo izquierdo. Todas las mediciones se realizaron en diástole, la cual se determinó en función de la posición de las válvulas cardíacas.

4. Volúmenes: para la determinación de los volúmenes ventriculares se emplearon ecotomogramas idénticos a los utilizados para la medición de los diámetros ventriculares, de donde se derivaron los diámetros ventriculares, aplicando para el cálculo de los mismos la fórmula cúbica, modificada por Teichholz ( $7 \times$ (DD) $3 / 2,4+$ DD y $7 \times$ (DS) $3 /$ $2,4+$ DS), donde DD corresponde a diámetro diastólico y DS a diámetro sistólico.

5. Índices de la fase de eyección: las medidas para la obtención de la fracción de acortamiento se realizaron en modo-M guiado a partir de una ima- gen del ventrículo izquierdo en eje corto, a nivel de las cuerdas tendinosas. El cursor de corte se colocó equidistante de cada grupo de cuerdas tendinosas. Para el cálculo se utilizó la formula \%AF = DDVI - DSVI / DDVI x 100 (\%AF: porcentaje de acortamiento fraccional, DDVI: diámetro diastólico del ventrículo izquierdo, DSVI: diámetro sistólico del ventrículo izquierdo).

Los valores de la Fracción de eyección se calcularon a partir de ecotomogramas idénticos a los utilizados para el cálculo de la fracción de acortamiento, mediante la fórmula FE = VDVI - VSVI / VDVI $x$ 100 (VDVI: volumen diastólico del ventrículo izquierdo, VSVI: volumen sistólico del ventrículo izquierdo). Para cada una de las determinaciones se emplearon imágenes diferentes.

Para el cálculo del porcentaje de engrosamiento de la pared posterior se utilizó la fórmula \%EFPP = ESPP - EDPP/EDPP y para el del tabique interventricular \%EFTI = ESTI - EDTI/ EDTI.

Para calcular la Velocidad de acortamiento circunferencial se empleó la fórmula Vac: VDVI-DSVI / DDVI x TEVI expresado el resultado en circ./seg.

La Separación septal del punto E se midió verticalmente, entre el punto E del ecograma mitral y la correspondiente porción del endocardio distal del tabique interventricular, a partir de una imagen en - eje corto a nivel de la válvula mitral y el cálculo para la obtención de la SSPE normalizado se realizó dividiendo su valor por el VDVI.

6. Intervalos sistólicos: la medición de los parámetros estudiados se realizó en modo-M guiado a partir de ecotomogramas registrados en eje corto, a nivel de la base cardíaca en el punto en el cual la arteria aorta se visualiza con forma trebolar y los bordes de las valvas valvulares son perfectamente identificables. El cursor de corte se colocó de tal manera que transectara la aorta en su punto medio. El PPEVI se midió desde la primera deflexión del complejo ventricular del electrocardiograma y la apertura de la válvula aórtica. El TEVI se midió entre la apertura y el cierre de la válvula aórtica. $\mathrm{La}$ SETVI se calculó sumando los valores del PPEVI y el TEVI y la relación PPEVI/TEVI efectuando el correspondiente cálculo.

Para todas las mediciones el valor computado resultó del promedio de cinco determinaciones efectuadas en ecotomogramas diferentes.

El procesamiento estadístico consistió en la obtención de las medidas resumen (valores máximo y mínimo, media, mediana, primer cuartil, tercer cuartil, desviación estándar y coeficiente de varia- 
ción). Asimismo se estudió la correlación entre la SSPE y el AF y la SSPEn y el AF, entre los intervalos sistólicos, la frecuencia cardíaca y el peso corporal, entre los diámetros ventriculares y el peso corporal, entre los volúmenes ventriculares y el peso corporal y entre los espesores parietal y septal y el peso corporal. Asimismo, en los casos en los cuales se encontró correlación significativa se realizó un estudio de regresión lineal.

\section{RESULTADOS}

Los resultados obtenidos para los distintos parámetros estudiados fueron los siguientes (ver tabla I):

\section{A) Medidas anatómicas}

1. Diámetro diastólico del ventrículo izquierdo (DDVI) $(\mathbf{n}=99)$. Media $=10,31 \mathrm{~cm}$; Desvío estándar $=1,17 \mathrm{~cm} ;$ Coeficiente de variación $=$ $11,38 \%$; Valor mínimo $=7,75 \mathrm{~cm}$; Valor máximo $=$ $13,80 \mathrm{~cm}$; Mediana $=10,15 \mathrm{~cm}$; Primer cuartil $=$ $9,60 \mathrm{~cm}$; Tercer cuartil $=11,05 \mathrm{~cm}$. Coeficiente de Pearson (r) para DDVI / Peso: 0,4731; Probabilidad asociada (p) para DDVI / Peso: 0,0000

2. Diámetro sistólico del ventrículo izquierdo (DSVI) $(\mathrm{n}=99)$. Media $=6,16 \mathrm{~cm}$; Desvío estándar $=0,98 \mathrm{~cm}$; Coeficiente de variación $=15,90 \%$; Valor mínimo $=4,45 \mathrm{~cm}$; Valor máximo $=9,75 \mathrm{~cm}$; Mediana $=6,05 \mathrm{~cm}$; Primer cuartil $=5,55 \mathrm{~cm}$; Tercer cuartil $=6,60 \mathrm{~cm}$; Coeficiente de Pearson (r) para DSVI / Peso: 0,3171; Probabilidad asociada (p) para DSVI / Peso: 0,0014.

Los resultados indican que las variables estudiadas están significativamente correlacionadas con el peso corporal $(p<0.01)$. Regresión lineal en el peso: coeficiente de regresión DDVI: 0,0092; DSVI: 0,0052 . Probabilidad asociada al coeficiente de regresión (p) = DDVI: 0,0004; DSVI: 0,0014 Coeficiente de determinación $\left(\mathrm{R}^{2}\right)=$ DDVI: 0,1356 ; DSVI: 0,1005 .

Si bien las regresiones resultan significativas ( $p<$ 0.01), el hecho de que el coeficiente de determinación, tomado como medida de ajuste, resulte bajo (menor al $33 \%$ en todos los casos), indica un grado de dispersión que no justifica la utilización del peso como variable predictora. Tampoco se observó tendencia no lineal entre las variables en estudio y el peso.

3. Espesor diastólico de la pared posterior del ventrículo izquierdo $($ EDPP) $(n=76)$. Media $=$
$2,16 \mathrm{~cm}$; Desvío estándar $=0,46 \mathrm{~cm}$; Coeficiente de variación $=21,17 \%$; Valor mínimo $=1,25 \mathrm{~cm}$; Valor máximo $=3,25 \mathrm{~cm}$; Mediana $=2,10 \mathrm{~cm}$; Primer cuartil $=1,75 \mathrm{~cm}$; Tercer cuartil $=2,50 \mathrm{~cm}$.

4. Espesor sistólico de la pared posterior del ventrículo izquierdo $($ ESPP) $(n=76)$. Media $=$ $3,57 \mathrm{~cm}$; Desvío estándar $=0,56 \mathrm{~cm}$; Coeficiente de variación $=15,72 \%$; Valor mínimo $=2,10 \mathrm{~cm}$; Valor máximo $=4,70 \mathrm{~cm}$; Mediana $=3,65 \mathrm{~cm}$; Primer cuartil $=3,25 \mathrm{~cm}$; Tercer cuartil $=3,95 \mathrm{~cm}$.

5. Espesor diastólico del tabique interventricular $($ EDTI $)(\mathbf{n}=\mathbf{8 7})$. Media $=2,57 \mathrm{~cm}$; Desvío estándar $=0,45 \mathrm{~cm}$; Coeficiente de variación $=$ 17,43\%; Valor mínimo $=1,65 \mathrm{~cm}$; Valor máximo $=$ $3,65 \mathrm{~cm}$; Mediana $=2,60 \mathrm{~cm}$; Primer cuartil $=$ $2,22 \mathrm{~cm}$; Tercer cuartil $=2,90 \mathrm{~cm}$.

6. Espesor sistólico del tabique interventricular $($ ESTI) $(\mathbf{n}=\mathbf{8 7})$. Media $=4,07 \mathrm{~cm}$; Desvío estándar $=0,55 \mathrm{~cm}$; Coeficiente de variación $=$ $13,59 \%$; Valor mínimo $=2,70 \mathrm{~cm}$; Valor máximo $=$ $5,35 \mathrm{~cm}$; Mediana $=4,10 \mathrm{~cm}$; Primer cuartil $=$ $3,70 \mathrm{~cm}$; Tercer cuartil $=4,40 \mathrm{~cm}$.

Se investigó la correlación de cada uno de los espesores con el peso corporal. Coeficiente de Pearson (r) = EDPP: 0,1587; ESPP: 0,3372; EDTI: 0,2004; ESTI: 0,4255. Probabilidad asociada $(\mathrm{p})=$ EDPP: 0,1709; ESPP: 0,0029; EDTI: 0,0627; ESTI: 0,0000.

Las variables ESPP y ESTI se encuentran significativamente correlacionadas con el peso corporal ( $p<0.01$ ), mientras que las variables EDPP y EDTI no están significativamente correlacionadas con el peso ( $p>0.01$ ). Regresión lineal en el peso: coeficiente de regresión ESPP: 0,0032; ESTI: 0,0040. Probabilidad asociada al coeficiente de regresión (p)= ESPP: 0,0029; ESTI: 0,0000. Coeficiente de determinación $\left(\mathrm{R}^{2}\right)=$ ESPP: 0.1137 ; ESTI: 0.1810 .

Si bien las dos regresiones resultan significativas $(p<0.01)$, el hecho de que el coeficiente de determinación, tomado como medida de ajuste, resulte bajo (menor al 33\% en ambos casos), indicó un grado de dispersión de valores que no justifica la utilización del peso como variable predictora. Tampoco se observa una tendencia no lineal entre las variables en estudio y el peso.

7. Diámetro diastólico del atrio izquierdo (DDAI) $(\mathbf{n}=38)$. Media $=9,01 \mathrm{~cm}$; Desvío Estándar $=0,92 \mathrm{~cm}$; Coeficiente de variación $=10,2 \%$; Valor mínimo $=7,35 \mathrm{~cm}$; Valor máximo $=10,75$ $\mathrm{cm}$; Mediana $=9,25 \mathrm{~cm}$; Primer cuartil $=8,35 \mathrm{~cm}$; Tercer cuartil $=9,78 \mathrm{~cm}$

8. Diámetro diastólíco del Anillo Aórtico (DDAAo) $(\mathrm{n}=\mathbf{2 6})$. Media: $6,16 \mathrm{~cm}$; Desviación es- 
tándar: $1,10 \mathrm{~cm}$; Coeficiente de variación: 17,67\%; Mediana: $6,10 \mathrm{~cm}$; Valor mínimo: $4,70 \mathrm{~cm}$; Valor máximo: $8,05 \mathrm{~cm}$; Primer cuartil: $5,20 \mathrm{~cm}$; Tercer cuartil: $7,05 \mathrm{~cm}$.

9. Diámetro diastólico del seno de Valsalva (DDSV) $(\mathbf{n}=32)$. Media: 7,28 cm; Desviación estándar: 0,90 cm; Coeficiente de variación: 12,39\%; Mediana: $6,97 \mathrm{~cm}$; Valor mínimo: $6,30 \mathrm{~cm}$; Valor máximo: $9,8 \mathrm{~cm}$; Primer cuartil: $6,70 \mathrm{~cm}$; Tercer cuartil: $7,61 \mathrm{~cm}$.

10. Diámetro diastólico de la raíz aórtica (DDRAo) $(\mathbf{n}=\mathbf{2 8})$. Media: $5,69 \mathrm{~cm}$; Desviación estándar: $0,55 \mathrm{~cm}$; Coeficiente de variación: $9,62 \%$; Mediana 5,63; Valor mínimo: $5,00 \mathrm{~cm}$, Valor máximo: $6,60 \mathrm{~cm}$; Primer cuartil: $5,21 \mathrm{~cm}$; Tercer cuartil: $6,21 \mathrm{~cm}$.

11. Área diastólica del anillo aórtico (ADAAo) $(\mathbf{n}=22)$. Media: $40,30 \mathrm{~cm}^{2}$; Desviación estándar: $6,53 \mathrm{~cm}^{2}$; Coeficiente de variación: $16,21 \%$; Mediana: $39,37 \mathrm{~cm}^{2}$; Valor mínimo: $32,33 \mathrm{~cm}^{2}$; Valor máximo: $61,44 \mathrm{~cm}^{2}$; Primer Cuartil: $35,50 \mathrm{~cm}^{2}$; Tercer cuartil: $42,86 \mathrm{~cm}^{2}$

12. Perímetro diastólico anillo aórtico (PDAAo) $(\mathbf{n}=22)$. Media: $23,38 \mathrm{~cm}$; Desviación estándar: $1,83 \mathrm{~cm}$; Coeficiente de variación: $7,84 \%$; Mediana: 23,32 cm; Valor mínimo: $20,90 \mathrm{~cm}$; Valor máximo: $28,95 \mathrm{~cm}$; Primer cuartil: $21,85 \mathrm{~cm}$; Tercer cuartil: $24,25 \mathrm{~cm}$.

13. Volumen diastólico del ventrículo izquierdo (VDVI) $(\mathbf{n}=97)$. Media $=615,50 \mathrm{~cm} 3$; Desvío estándar $=156,63 \mathrm{~cm} 3$; Coeficiente de variación $=25,45 \%$; Valor mínimo $=320,90 \mathrm{~cm} 3$; Valor máximo $=1.135,60 \mathrm{~cm} 3 ;$ Mediana $=$ $595,91 \mathrm{~cm} 3$; Primer cuartil $=516,20 \mathrm{~cm} 3$; Tercer cuartil $=702,20 \mathrm{~cm} 3$. Coeficiente de Pearson (r) VDVI / Peso: 0,4712; Probabilidad asociada (p) VDVI / Peso: 0,0000.

14. Volumen sistólico del ventrículo izquierdo (VSVI) $(\mathbf{n}=97)$. Media $=200,66 \mathrm{~cm} 3$; Desvío estándar $=77,71 \mathrm{~cm} 3$; Coeficiente de variación $=38.73 \%$; Valor mínimo $=90,18 \mathrm{~cm} 3$; Valor máximo $=533,99 \mathrm{~cm} 3$; Mediana $=183,75 \mathrm{~cm} 3$; Primer cuartil $=150,52 \mathrm{~cm} 3$; Tercer cuartil $=232,49 \mathrm{~cm} 3$. Coeficiente de Pearson (r) VSVI / Peso: 0,3163. Probabilidad asociada (p) VSVI / Peso: 0,0016.

En función de los resultados obtenidos surge que los volúmenes ventriculares están significativamente correlacionados con el peso corporal $(p<0,01)$. Regresión lineal en el peso: coeficiente de regresión VDVI: 1,2208 ; VSVI: 0,4066 . Probabilidad asociada al coeficiente de regresión (p) VDVI / Peso: 0,0007; VSVI/ Peso: 0,0016. Coeficiente de deter- minación (R2) VDVI / peso: 0,1291; VSVI / Peso: 0,1000 .

\section{B) ÍNDICES DE LA FASE DE EYECCIÓN}

1. Porcentaje de acortamiento fraccional. $(\% \mathbf{A F})(\mathbf{n}=101)$ Media = 39,62\%; Desviación estándar $=5,30 \%$; Coeficiente de variación $=13,39 \%$; Valor mínimo $=27 \%$; Valor máximo $=51 \%$; Mediana $=40 \%$; Primer cuartil $=36 \%$; Tercer cuartil $=44 \%$.

2. Fracción de eyección. (FE) $(n=101)$. Media $=67,40 \%$; Desviación estándar $=6,81 \%$; Coeficiente de variación $=10,11 \%$; Valor mínimo $=$ $50,40 \%$; Valor máximo $=80,20 \%$; Mediana $=$ $68,05 \%$; Primer cuartil $=63,05$; Tercer cuartil $=$ $72,76 \%$.

3. Porcentaje de engrosamiento fraccional del tabique interventricular. (\%EFTI) $(n=42)$. Media $=35 \%$; Desviación estándar $=9,75 \%$; Coeficiente de variación $=27,87 \%$; Valor mínimo $=13 \%$; Valor máximo $=57 \%$; Mediana: $36 \%$; Primer cuartil: $30,5 \%$; Tercer cuartil: $41,25 \%$.

4. Porcentaje de engrosamiento fraccional de la pared posterior del ventrículo izquierdo. (\%EFPP) $(\mathbf{n}=32)$. Media $=38,97 \%$; Desviación estándar $=11,44 \%$; Coeficiente de variación $=$ 29,37\%; Valor mínimo $=16 \%$; Valor máximo $=$ $71 \%$; Mediana: 39,5\%; Primer cuartil: 33,25\%; Tercer cuartil: $44,75 \%$

5. Velocidad de acortamiento circunferencial (VAC) (n = 39). Media = 0,77 circ. $/ \mathrm{seg}$; Desviación estándar $=0,18 \mathrm{circ} . / \mathrm{seg}$; Coeficiente de variación $=33,31 \%$; Valor mínimo $=0,45$ circ. $/ \mathrm{seg}$; Valor máximo $=1,21 \mathrm{circ} . / \mathrm{seg}$ Mediana: $0,75 \mathrm{circ} /$ seg; Primer cuartil: 0,13 circ/seg;Tercer cuartil: $0,87 \mathrm{circ} / \mathrm{seg}$.

6. Separación septal del punto E. (SSPE) $(n=$ 37). Media $=1,55 \mathrm{~cm}$; Desviación estándar $=$ $0,47 \mathrm{~cm}$; Coeficiente de variación $=30,51 \%$. Mediana $=1,63 \mathrm{~cm}$; Valor mínimo $=0,4 \mathrm{~cm}$; Valor máximo $=2,4 \mathrm{~cm}$. Primer cuartil: $1,25 \mathrm{~cm}$; Tercer cuartil: $1,87 \mathrm{~cm}$. No se observó correlación significativa entre la SSPE y el \%AF (P-value: 0,3303, r:0,1671 ).

7. Separación septal del punto $E$ normalizada. $($ SSPEn) $(\mathbf{n}=\mathbf{3 4})$. Media $=0,15 \mathrm{~cm}$; Desviación estándar $=0,05 \mathrm{~cm}$; Coeficiente de variación $=32,30 \%$; Valor mínimo $=0,04 \mathrm{~cm}$; Valor máximo $=0,24 \mathrm{~cm}$; Mediana: $0,17 \mathrm{~cm}$. No se observo correlación significativa entre la SSPE normalizada y el \% AF (P-value: 0,4264; r: 0,1410. 


\section{C) LNTERVALOS SISTÓLICOS}

1. Período pre-eyectivo del ventrículo izquierdo $($ PPE $)(\mathbf{n}=\mathbf{8 7})$. Media = 0,072 seg; Desviación estándar $=0,012 \mathrm{seg}$; Coeficiente de variación $=$ 16,6\%; Mediana $=0,07$ seg; Valor mínimo $=0,05$ seg; Valor máximo $=0,09 \mathrm{seg}$; Primer cuartil $=0,06$ seg; Tercer cuartil $=0,08 \mathrm{seg}$. Coeficiente de correlación PPE/Frecuencia cardíaca $=-0,1410(\mathrm{p}:-$ $0,1954)$; Coeficiente de correlación PPE/ Peso $=$ 0,2667 (p: 0,1055).

2. Tiempo de eyección del ventrículo izquierdo $($ TEVI $)(\mathbf{n}=\mathbf{8 7})$. Media = 0,529 seg; Desviación estándar $=0,103 \mathrm{seg}$; Coeficiente de variación $=19,4 \%$; Mediana $=0,51 \mathrm{seg} ;$ Valor mínimo $=0,45$ seg; Valor máximo = 0,60 seg; Primer cuartil $=0,45$; Tercer cuartil $=0,60 \mathrm{seg}$. Coeficiente de correlación TEVI/ Frecuencia cardíaca $=-0,9247$ (p: 0,0000); Coeficiente de correlación TEVI/ Peso = 0,2803, (p: 0,0973). Coeficiente de regresión TEVI/FC: 0,0129, (p): 0,0000. Coeficiente de determinación: 0,8551

3. Sístole electromecánica total $($ SET) $(n=$ 87). Media $=0,598 \mathrm{seg}$; Desviación estándar $=$ $0,104 \mathrm{seg}$; Coeficiente de correlación $=17,4 \%$; Mediana $=0,58 \mathrm{seg}$; Valor mínimo $=0,39 \mathrm{seg}$; Valor máximo $=0,86 \mathrm{seg}$; Primer cuartil $=0,52 \mathrm{seg}$; Tercer cuartil $=0,68 \mathrm{seg}$. Coeficiente de correlación SET/ Frecuencia cardíaca $=-0,9174,(p: 0,0000$ ); Coeficiente de correlación SET/Peso $=0,1758$, (p): 0,3047. Coeficiente de regresión SET/FC: -0,0130; (p): 0,0000. Coeficiente de determinación: 0,8417

4. Relación Período pre-eyectivo / Tiempo de eyección. (PPE/TEVI) $(\mathbf{n}=\mathbf{8 7})$. Media $=0,140$; Desviación estándar $=0,033$; Coeficiente de correlación $=23,7 \%$; Mediana $=0,130$; Valor mínimo $=$ 0,09 ; Valor máximo $=0,24$; Primer cuartil $=0,11$; Tercer cuartil $=0,16$. Coeficiente de correlación PPE-TEVI/Frecuencia cardíaca $=0,6702,(\mathrm{p}$ : 0,0000 ); Coeficiente de correlación PPE-TEVI/ Peso $=0,0004$, (p: 0,9982). Coeficiente de regresión PPE/TEVI con FC.: 0,0030; (p): 0,0000. Coeficiente de determinación: 0,4491.

La Tablas I y II resumen los valores obtenidos en el presente estudio

\section{COMENTARIOS}

En ecocardiografía la exactitud de las mediciones depende de varios factores, considerando como los más importantes la correcta elección del ecoto- mograma, la adhesión a alguno de los principios de medición universalmente aceptados y la repetición de las mediciones para obtener, como cifra definitiva, el promedio de varias determinaciones $[14,16$, $33,35]$.

El problema más serio que se encuentra cuando se analizan los valores de referencia ecocardiográficos publicados para el caballo es la falta de indicación o la poco precisa especificación de la metodología empleada para la recolección de los mismos $[36,53,59,61]$.

Los valores pueden variar, además, en función de la población elegida para realizar el estudio y el estado de los individuos que la conforman, hecho que tampoco es especificado con claridad en muchas publicaciones.

Otro aspecto poco tenido en cuenta cuando se realizan estudios para establecer valores de referencia es el grado de entrenamiento que presentan los animales empleados, dado que existe amplia información sobre el efecto directo que tiene el entrenamiento sobre los parámetros ecocardiográficos, al igual que el desentrenamiento $[40,44,48]$.

En el presente estudio hemos cuidado de establecer con precisión la metodología empleada y definido adecuadamente la muestra utilizada para la investigación.

De los resultados del presente estudio surgen coincidencias y diferencias entre los valores obtenidos y los publicados por otros investigadores. Se entiende que dichas diferencias son debidas a alguno o a todos los factores mencionados precedentemente como posibles fuentes de error.

Descontando que el operador cuenta con la calidad técnica para la recolección de los datos, se considera que el punto más importante a tener en cuenta para futuros estudios sobre el tema es la selección de la muestra a estudiar, atendiendo en especial al biotipo de animales utilizados, al uso $\mathrm{y}$, fundamentalmente, al grado y tipo de entrenamiento que presentan en el momento del estudio.

Son escasas las referencias que existen sobre el estudio de los volúmenes ventriculares izquierdos en el caballo y menos aun sobre la utilización de las distintas fórmulas para su cálculo a través de la ecocardiografía [32, 62]. Es bien conocida la dificultad que existe en la especie que nos ocupa para obtener imágenes en eje largo donde pueda representarse el ventrículo entero y mostrar el ápex cardíaco verdadero. Por ello, todas las fórmulas para calcular los volúmenes ventriculares en las cuales deba emplearse el valor del eje largo (Hemielipse, 
TABLA I

VALORES ECOCARDIOGRÁFICOS

\begin{tabular}{|c|c|c|c|c|c|c|c|c|c|}
\hline Parámetro & $n$ & Media & $D . E$. & C.V. & Mediana & V.min. & V.máx. & $I^{e r}$ cuart & $3^{e r}$ cuar. \\
\hline DDVI & 99 & $10,31 \mathrm{~cm}$ & $1,17 \mathrm{~cm}$ & $11,38 \%$ & $10,15 \mathrm{~cm}$ & $7,75 \mathrm{~cm}$ & $13,80 \mathrm{~cm}$ & $9,60 \mathrm{~cm}$ & $11,05 \mathrm{~cm}$ \\
\hline DSVI & 99 & $6,16 \mathrm{~cm}$ & $0,98 \mathrm{~cm}$ & $15,90 \mathrm{~cm}$ & $6,05 \mathrm{~cm}$ & $4,45 \mathrm{~cm}$ & $9,75 \mathrm{~cm}$ & $5,55 \mathrm{~cm}$ & $6,60 \mathrm{~cm}$ \\
\hline EDPP & 76 & $2,16 \mathrm{~cm}$ & $0,46 \mathrm{~cm}$ & $21,17 \%$ & $2,10 \mathrm{~cm}$ & $1,25 \mathrm{~cm}$ & $3,25 \mathrm{~cm}$ & $1,75 \mathrm{~cm}$ & $2,50 \mathrm{~cm}$ \\
\hline ESPP & 76 & $3,57 \mathrm{~cm}$ & $0,56 \mathrm{~cm}$ & 15,725 & $3,65 \mathrm{~cm}$ & $2,10 \mathrm{~cm}$ & $4,70 \mathrm{~cm}$ & $3,25 \mathrm{~cm}$ & $3,95 \mathrm{~cm}$ \\
\hline EDTI & 87 & $2,57 \mathrm{~cm}$ & $0,45 \mathrm{~cm}$ & $17,43 \%$ & $2,60 \mathrm{~cm}$ & $1,65 \mathrm{~cm}$ & $3,65 \mathrm{~cm}$ & $2,22 \mathrm{~cm}$ & $2,90 \mathrm{~cm}$ \\
\hline ESTI & 87 & $4,07 \mathrm{~cm}$ & $0,55 \mathrm{~cm}$ & $13,59 \%$ & $4,10 \mathrm{~cm}$ & $2,70 \mathrm{~cm}$ & $5,35 \mathrm{~cm}$ & $3,70 \mathrm{~cm}$ & $4,40 \mathrm{~cm}$ \\
\hline DDAI & 38 & $9,01 \mathrm{~cm}$ & $0,92 \mathrm{~cm}$ & $10,20 \mathrm{~cm}$ & $9,25 \mathrm{~cm}$ & $7,35 \mathrm{~cm}$ & $10,75 \mathrm{~cm}$ & $8,35 \mathrm{~cm}$ & $9,78 \mathrm{~cm}$ \\
\hline DDAAo & 26 & $6,16 \mathrm{~cm}$ & $1,10 \mathrm{~cm}$ & $17,67 \%$ & $6,10 \mathrm{~cm}$ & $4,70 \mathrm{~cm}$ & $8,05 \mathrm{~cm}$ & $5,20 \mathrm{~cm}$ & $7,05 \mathrm{~cm}$ \\
\hline DDSV & 32 & $7,28 \mathrm{~cm}$ & $0,90 \mathrm{~cm}$ & $12,39 \%$ & $6,97 \mathrm{~cm}$ & $6,30 \mathrm{~cm}$ & $9,80 \mathrm{~cm}$ & $6,70 \mathrm{~cm}$ & $7,61 \mathrm{~cm}$ \\
\hline DDRAo & 28 & $5,69 \mathrm{~cm}$ & $0,55 \mathrm{~cm}$ & $9,62 \%$ & $5,63 \mathrm{~cm}$ & $5,00 \mathrm{~cm}$ & $6,60 \mathrm{~cm}$ & $5,21 \mathrm{~cm}$ & $6,21 \mathrm{~cm}$ \\
\hline ADAAo & 22 & $40,30 \mathrm{~cm}^{2}$ & $6,53 \mathrm{~cm}^{2}$ & $16,21 \%$ & $39,37 \mathrm{~cm}^{2}$ & $32,33 \mathrm{~cm}^{2}$ & $61,44 \mathrm{~cm}^{2}$ & $35,50 \mathrm{~cm}^{2}$ & $42,86 \mathrm{~cm}^{2}$ \\
\hline PDAAO & 22 & $23,38 \mathrm{~cm}$ & $1,83 \mathrm{~cm}$ & $7,84 \%$ & $23,32 \mathrm{~cm}$ & $20,90 \mathrm{~cm}$ & $28,95 \mathrm{~cm}$ & $21,85 \mathrm{~cm}$ & $24,25 \mathrm{~cm}$ \\
\hline VDVI & 97 & $615,50 \mathrm{ml}$ & $156,63 \mathrm{ml}$ & $25,45 \%$ & $595,91 \mathrm{ml}$ & $320,90 \mathrm{ml}$ & $1135,6 \mathrm{ml}$ & $516,20 \mathrm{ml}$ & $702,20 \mathrm{ml}$ \\
\hline VSVI & 97 & $200,66 \mathrm{ml}$ & $77,71 \mathrm{ml}$ & $38,73 \%$ & $183,75 \mathrm{ml}$ & $90,18 \mathrm{ml}$ & $533,99 \mathrm{ml}$ & $150,52 \mathrm{ml}$ & $232,49 \mathrm{ml}$ \\
\hline$\% \mathrm{AF}$ & 101 & $39,62 \%$ & $5,30 \%$ & $13,39 \%$ & $40,00 \%$ & $27 \%$ & $51 \%$ & $36,00 \%$ & $44,00 \%$ \\
\hline $\mathrm{FE}$ & 101 & $67,40 \%$ & $6,81 \%$ & $10,11 \%$ & $68,05 \%$ & $50,40 \%$ & $80,20 \%$ & $63,05 \%$ & $72,76 \%$ \\
\hline \%EFPP & 32 & $38.97 \%$ & $11,44 \%$ & $29,37 \%$ & $39,50 \%$ & $16 \%$ & $71 \%$ & $33,25 \%$ & $44,75 \%$ \\
\hline$\%$ EFTI & 42 & $35 \%$ & $9,75 \%$ & $27,87 \%$ & $36,00 \%$ & $13 \%$ & $57 \%$ & $30,50 \%$ & $41,25 \%$ \\
\hline $\mathrm{Vac}$ & 39 & $0,77 \mathrm{c} / \mathrm{seg}$ & $0,18 \mathrm{c} / \mathrm{seg}$ & $33,31 \%$ & $0,75 \mathrm{c} / \mathrm{seg}$ & $0,45 \mathrm{c} / \mathrm{seg}$ & $1,21 \mathrm{c} / \mathrm{seg}$ & $0,13 \mathrm{c} / \mathrm{seg}$ & $0,87 \mathrm{c} / \mathrm{seg}$ \\
\hline SSPE & 37 & $1,55 \mathrm{~cm}$ & $0,47 \mathrm{~cm}$ & $30,51 \%$ & $1,63 \mathrm{~cm}$ & $0,40 \mathrm{~cm}$ & $2,40 \mathrm{~cm}$ & $1,25 \mathrm{~cm}$ & $1,87 \mathrm{~cm}$ \\
\hline SSPEn & 34 & 0,15 & 0,05 & $32,30 \%$ & 0,17 & 0,04 & 0,24 & - & - \\
\hline PPE & 87 & $0,072 \mathrm{seg}$ & $0,012 \mathrm{seg}$ & $16,6 \%$ & $0,07 \mathrm{seg}$ & $0,05 \mathrm{seg}$ & $0,09 \mathrm{seg}$ & $0,06 \mathrm{seg}$ & 0,08 seg \\
\hline TEVI & 87 & $0,529 \mathrm{seg}$ & $0,103 \mathrm{seg}$ & $19,4 \%$ & $0,51 \mathrm{seg}$ & $0,45 \mathrm{seg}$ & $0,60 \mathrm{seg}$ & $0,45 \mathrm{seg}$ & $0,60 \mathrm{seg}$ \\
\hline SET & 87 & $0,598 \mathrm{seg}$ & $0,104 \mathrm{seg}$ & $17,4 \%$ & $0,58 \mathrm{seg}$ & $0,39 \mathrm{seg}$ & 0,86 seg & $0,52 \mathrm{seg}$ & $0,68 \mathrm{seg}$ \\
\hline PPE/TEVI & 87 & 0,140 & 0,033 & $23,7 \%$ & 0,130 & 0,09 & 0,24 & 0,11 & 0,16 \\
\hline
\end{tabular}

D.E.: desviación estándar; C.V.: coeficiente de variación; V. min.: valor mínimo; V. máx.:Valor máximo; $1^{\mathrm{er}}$ cuar.: 1er cuartil; $3^{\text {er }}$ cuar.: $3^{\text {er }}$ cuartil; DDVI: diámetro diastólico del ventrículo izquierdo; DSVI: diámetro sistólico del ventrículo izquierdo; EDPP: espesor diastólico de la pared posterior del ventrículo izquierdo; ESPP: espesor sistólico de la pared posterior del ventrículo izquierdo; EDTI: espesor diastólico del tabique interventricular; ESTI: espesor sistólico del tabique interventricular; DDAI: diámetro diastólico del atrio izquierdo; DDAAo: diámetro diastólico del anillo aórtico; DDSV: diámetro diastólico del seno del Valsalva; DDRAo: diámetro diastólico de la raíz aórtica; ADAAo: área diastólico del anillo aórtico; PDAAo: perímetro diastólico del anillo aórtico; VDVI: volumen diastólico del ventrículo izquierdo; VSVI: volumen sistólico del ventrículo izquierdo; \%AF: \% acortamiento fraccional; FE: fracción de eyección; \%EFPP: $\%$ engrosamiento fraccional de la pared posterior del ventrículo izquierdo; \%EFTI: \% engrosamiento fraccional del tabique interventricular; Vac: velocidad de acortamiento circunferencial; SSPE: separación septal del punto E; SSPEn: separación septal del punto E normalizada; PPE: período pre-eyectivo del ventrículo izquierdo; TEVI: tiempo de eyección del ventrículo izquierdo; SET: sístole electromecánica total; PPE/TEVI: relación período eyectivo / tiempo de eyección ventricular izquierda.

integral de Simpson) están vedadas para el caballo. En el presente estudio se ha empleado la fórmula cúbica modificada por Teichholz por ser la más ampliamente utilizada en el hombre y en el perro y considerada por algunos autores $[32,57]$ como una de las más adecuadas para su empleo en el equino

Está demostrado para humanos $[9,16,20]$ y en el perro $[8,9]$ que existen parámetros que se correlacionan con el peso o la superficie corporal (tamaño de cámaras, espesores parietal y septal, volúmenes ventriculares), mientras que otros son independientes (básicamente los índices ecocardiográficos). En una publicación [31] no encontraron correlación de los diámetros ventriculares con el peso. Nuestros resultados indican la existencia de una correlación débil entre el peso corporal y los diámetros y volúmenes ventriculares (ver Tabla II). Sin embargo, los resultados de la regresión no permitieron establecer una tendencia lineal o no lineal que posibilite desarrollar una gráfica Peso/Diámetros o Peso/ Volúmenes. Teniendo en cuenta lo mencionado parecería inapropiado promediar los valores de los diámetros y volúmenes ventriculares.

Sin embargo, cuando se analiza la bibliografía se hace evidente que la mayoría de los investigadores publican promediados los valores de los diá- 
TABLA II

VALORES DE LOS ÍNDICES DE CORRELACIÓN Y REGRESIÓN DE ALGUNOS DE LOS PARÁMETROS ESTUDIADOS

\begin{tabular}{|c|c|c|c|c|c|}
\hline Parámetros & $\begin{array}{c}\text { Coeficiente } \\
\text { de Correlación }\end{array}$ & $\begin{array}{c}\text { Probabilidad Asociada } \\
\text { al Coeficiente de Regresión }\end{array}$ & $\begin{array}{l}\text { Coeficiente } \\
\text { de Regresión }\end{array}$ & $\begin{array}{l}\text { Probabilidad } \\
\text { Asociada }\end{array}$ & $\begin{array}{c}\text { Coeficiente } \\
\text { de Determinación }\end{array}$ \\
\hline DDVI / Peso & 0,4731 & 0,0000 & 0,0092 & 0,0004 & 0,1356 \\
\hline DSVI / Peso & 0,3171 & 0,0014 & 0,0052 & 0,0014 & 0,1005 \\
\hline VDVI / Peso & 0,4712 & 0,0000 & 1,2208 & 0,0007 & 0,1291 \\
\hline VSVI / Peso & 0,3163 & 0,0016 & 0,4066 & 0,0016 & 0,1000 \\
\hline EDPP / Peso & 0,1587 & 0,1709 & - & - & - \\
\hline ESPP / Peso & 0,3372 & 0,0029 & 0,0032 & 0,0029 & 0,1137 \\
\hline EDTI / Peso & 0,2004 & 0,0627 & - & - & - \\
\hline ESTI / Peso & 0,4255 & 0,0000 & 0,0040 & 0,0000 & 0,1810 \\
\hline $\mathrm{SSPE} / \% \mathrm{AF}$ & $-0,1671$ & 0,3303 & - & - & - \\
\hline $\mathrm{PPE} / \mathrm{FC}$ & $-0,1410$ & 0,1954 & - & - & - \\
\hline PPE / Peso & 0,2667 & 0,1055 & - & - & - \\
\hline TEVI / FC & $-0,9247$ & 0,0000 & $-0,0129$ & 0,0000 & 0,8551 \\
\hline TEVI / Peso & 0,2803 & 0,0973 & - & - & - \\
\hline SET / FC & $-0,9174$ & 0,0000 & $-0,0130$ & 0,0000 & 0,8417 \\
\hline SET / Peso & 0,1758 & 0,3047 & - & - & - \\
\hline PPE-TEVI / FC & 0,6702 & 0,0000 & 0,0030 & 0,0000 & 0,4491 \\
\hline PPE-TEVI / Peso & 0,0004 & 0,9982 & - & - & - \\
\hline
\end{tabular}

metros ventriculares del caballo, obtenidos de animales con un amplio rango de pesos, por lo que no parecería cuestionable, en el caballo, promediar estos valores, más si se tiene en cuenta que es prácticamente imposible trazar una línea de regresión por la amplia dispersión de valores (Tabla II) $[8,38,41]$.

Los espesores parietal y septal están entre los parámetros que también se encuentran relacionados con el peso o superficie corporal $[8,17,18,21,34$, $36,49,60$ ] y por lo tanto, al igual que para el caso de los diámetros ventriculares, podría considerarse incorrecto promediar sus valores para amplios rangos de pesos corporales.

Sin embargo, son muy escasas las publicaciones que hacen referencia a la existencia o no, en el caballo, de correlación entre el peso corporal y los espesores septal y parietal y su grado. En una publicación [31] tampoco pudieron encontrar correlación entre estos parámetros y el peso corporal. Los resultados aquí presentados muestran la existencia de una correlación positiva mediana entre el peso corporal y el ESPP y entre el peso corporal y el ESTI, mientras que las otras dos variables no correlacionaron significativamente con el peso (Tabla II).

El porcentaje de acortamiento fraccional y la fracción de eyección sistólica son dos de los índices utilizados con más frecuencia para evaluar la función global del ventrículo izquierdo $[2,3,12,29$, $55,56]$. El porcentaje de acortamiento fraccional re- presenta el porcentaje o fracción del diámetro ventricular en que se acorta el ventrículo durante la sístole, mientras que la fracción de eyección representa la fracción de volumen diastólico final que se eyecta en cada sístole; por expresar ambos índices funcionales cambios porcentuales, su valores no son influenciados por el peso, altura o superficie corporal, razón por la cual cada especie presenta un valor normal característico, independientemente del tamaño corporal individual [8].

El empleo clínico de cada uno de estos índices presenta ventajas y desventajas [16]. El porcentaje de acortamiento fraccional es fácil de determinar y que su valor es obtenido a partir de mediciones lineales (diámetros ventriculares), reduciéndose así los errores derivados de elevar al cubo dichos valores, como ocurre para el caso del cálculo de la fracción de eyección, donde es necesario asumir como ciertos algunos criterios geométricos respecto de la forma ventricular y aplicar distintas fórmulas correctivas para la obtención de los volúmenes ventriculares diastólico y sistólico.

A pesar de la incuestionable utilidad de los índices de la fase de eyección, tanto por su practicidad en la obtención como por su significado diagnóstico y pronóstico deben tenerse en cuenta ciertos aspectos que pueden modificar su valor.

Entre los más importantes está el estado de la pre y postcarga, así como también el valor de la fre- 
cuencia cardíaca, dado que estos factores influencian los resultados al determinar el punto de comienzo y finalización de la contracción ventricular. Se ha demostrado que la fracción de eyección varía en relación directa con el estado de la precarga e inversamente con el de la postcarga. No existen datos publicados para el caballo que indiquen la forma exacta en que se comporta la fracción de eyección en relación a la frecuencia cardíaca $[4,13,15$, $28,42,46,58]$.

La evaluación de la separación septal del punto E es importante dado que es otro parámetro de fácil determinación y de gran valor para evaluar el flujo transmitral, constituyéndose en una de las herramientas de elección para evaluar la función sistólica del ventrículo izquierdo [27].

La medición de los intervalos sistólicos a través de la ecocardiografía requiere la obtención de imágenes diagnósticas del ecograma aórtico y su correlación con el electrocardiograma [1].

El período pre-eyectivo del ventrículo izquierdo (PPEVI) mide el período de contracción isovolumétrica del ventrículo izquierdo, período durante el cual las válvulas mitral y aórtica se encuentran cerradas y el ventrículo incrementa su presión hasta igualar la presión diastólica aórtica, momento en que comienza el período de eyección; éste se inicia con la apertura de la válvula aórtica y culmina con su cierre, período conocido como tiempo de eyección del ventrículo izquierdo o período eyectivo (TEVI o Pey). La suma de ambos tiempos es lo que se conoce como sístole electromecánica total (SETVI) [27].

Estos índices, al igual que los de la fase de eyección, como ya se dijo, están fuertemente influenciados por la precarga, postcarga y la frecuencia cardíaca. Con el objeto de minimizar los efectos de este último parámetro sobre el TEVI se desarrolló la relación PPEVI / TEVI.

La velocidad de acortamiento circunferencial refleja la velocidad en que se reduce el tamaño del ventrículo izquierdo a nivel de su eje menor. Dicho de otra manera, este índice mide la magnitud del cambio de tamaño de la circunferencia ventricular en un determinado tiempo. Se ha mencionado que este índice es poco sensible a las variaciones agudas de la precarga y este hecho sumado a que dicho índice valora más la velocidad que el grado de acortamiento hace que se lo considere un parámetro más fidedigno de la contractilidad miocárdica, comportándose como un indicador precoz de los trastornos inotrópicos que desarrollan en los procesos cardíacos crónicos. Si bien la velocidad de acortamiento circunferencial es influenciada por la frecuencia cardíaca, hecho que dificulta las comparaciones entre valores, puede emplearse su valor corregido, el que se obtiene dividiendo el valor convencional por la raíz cuadrada del intervalo RR $[4,13,28,58]$.

El comportamiento de la pared posterior del ventrículo izquierdo y del septum interventricular a través del ciclo cardíaco ha sido estudiado de varias maneras $[14,16]$, entre las que se incluye el valor de la pendiente de la pared posterior, la excursión del septum interventricular y del endocardio posterior. Sin embargo el uso de la relación del comportamiento de ambas estructuras en sístole y en diástole ha ganado cierta popularidad en medicina veterinaria $[28,58]$ a pesar de que algunos investigadores [39] consideran que existe mucha variabilidad en los valores de estos índices como para considerarlos buenas herramientas para la evaluación de la función sistólica global. En este estudio la variabilidad fue mucho menor (ver Tabla I).

\section{RESUMEN}

Los autores realizaron un estudio para determinar distintos valores ecocardiográficos en el caba1lo. Evaluaron los siguientes parámetros: Diámetro Diastólico del Ventrículo Izquierdo (DDVI), Diámetro Sistólico del Ventrículo Izquierdo (DSVI), Espesor Diastólico del Tabique Interventricular (EDTI), Espesor Sistólico del Tabique Interventricular (ESTI), Espesor Diastólico de la Pared Posterior del ventrículo izquierdo (EDPP), Espesor Sistólico de la Pared Posterior del ventrículo izquierdo (ESPP), Diámetro Diastólico del Atrio Izquierdo (DDAI), Diámetro diastólico del Anillo Aórtico (DDAAo), Diámetro Diastólico del Seno de Valsalva (DDSV), Diámetro Diastólico de la Raíz Aórtica (DDRAo), Área Diastólica del Anillo Aórtico (ADAAo), Perímetro Diastólico del Anillo Aórtico (PDAAo), Volumen Diastólico del Ventrículo Izquierdo (VDVI) y Volumen Sistólico del Ventrículo Izquierdo (VDVI). Fracción de Acortamiento (FA), Fracción de Eyección (FE), Velocidad de acortamiento circunferencial (Vac), Engrosamiento Fraccional de la Pared Posterior del ventrículo izquierdo (EFPP), Engrosamiento Fraccional del Tabique Interventricular (EFTI), Separación Septal del Punto E (SSPE) y Separación Septal del Punto E normalizada (SSPEn), Período pre-eyectivo del ventrículo izquierdo (PPEVI), Tiempo de eyección del ventrículo izquierdo (TEVI), Sístole electromecáni- 
ca total del ventrículo izquierdo (SETVI) y relación PPEVI/TEVI.

El procesamiento estadístico consistió en la obtención de las medidas resumen (valores máximo y mínimo, media, mediana, primer cuartil, tercer cuartil, desviación estándar y coeficiente de variación). Asimismo se estudió la correlación entre la SSPE y el AF y la SSPEn y el AF, entre los intervalos sistólicos, la frecuencia cardíaca y el peso corporal, entre los diámetros ventriculares y el peso corporal, entre los volúmenes ventriculares y el peso corporal y entre los espesores parietal y septal y el peso corporal. Asimismo, en los casos en los cuales se encontró correlación significativa se realizó un estudio de regresión lineal.

Los valores resultantes de cada uno de los parámetros utilizados se encuentran en las tablas I y II del cuerpo del artículo.

\section{BIBLIOGRAFÍA}

1. ATKINS, C. E.; SNYDER, P. S. 1992. Systolic time intervals and their derivates for evaluation of cardiac function, $\mathrm{J}$. Vet Intern Med, 2: 55-63.

2. ANTANI, J. A.; H. H. WAYNE; W. J. KUZMAN. 1979. Ejection phase indexes by invasive and noninvasive methods: an apexocardiographic, echocardiographic and ventriculographic correlative study. Am. J. Cardiol. 43: 239-248.

3. BARAN, A. O.; G. J. ROGAL; N. C. NANDA. 1983. Ejection fraction determination without planimetry by two-dimensional echocardiography: a new method. J. Am Coll. Cardiol. 1471-1478.

4. BENZING, G.; STOCKERT, J.; NAVE, E.; KAPLAN, S. 1974. Evaluation of left ventricular performance: circunferential fiber shortening and tension. Circulation 49: 925.

5. Bertone, J. J.; Patนl, K. S.; Wingfield, W. E.; Boon, J. A. 1987. M-mode echocardiograms of endurance horses in the recovery phase of long-distance competition. Am. J. Vet. Res. 48: 1708-1712.

6. Bonagura, J. D.; HeRRING. D. S.; WeLKER, F. 1985. Echocardiography. Vet. Clin. N. Am. Equine Pract. 1, 311-333.

7. BonagurA, J. D. (ed). 1985. Symposium on cardiology (Horse). Vet. Clin. N. Am. Equine Pract, 1: 265-435.

8. BOON, J. 1998. Manual of Veterinary Echocardiography. $1^{\text {st }}$ Ed. Williams \& Wilkins.

9. BoON, J.; W. E. WINFIELD; C. W. MILLER. 1983. Echocardiographic indices in the normal dog. Vet. Radiol. 24 (5): 214-221.

10. CARLSTEN, J. C. 1987. Two-dimensional, real-time echocardiography in the horse. Vet. Radiol. 28: 76-87.

11. CARLSTEN, J. C. 1986. Imaging of the equine heart. An angiocardiographic and echocardiographic investigation, Thesis. Faculty of Veterinary Medicine, Swedish University of Agricultural Sciences, Uppsala, Sweden.

12. COOPER, R.; KARLINER, J. S.; O'ROURKE, R. A.; PETERSON, K. L.; LEOPOLD, G. R. 1972. Ultrasound determinations of mean fiber shortening rate in man. Am. J. Cardiol 29: 257.
13. COOPER, R. H.; O'RourKe, R. A.; Karliner, J. J.; PETERSEN, K. L.; LEOPOLD, G. R. 1972. Comparison of ultrasound and cineangiographic measurements of the mean rate of circunferential shortening in man. Circulation 46: 914.

14. CUESTA SILVA, M.; Boskis, P. J.; LERMAN, J. L.; BINFI LO, M. M., Torino, A.; SCATTIN, M. C.; BoskIS, B.; PERosio, A M. 1977. Ecocardiografía clínica. $1^{\text {st }}$ ed. Ed. El Ateneo, Buenos Aires.

15. DEVEREUX, R. B. 1991. Toward a more complete understanding of left ventricular afterload. JACC 17: 122-124.

16. FEIGENBAUN, H. 1995. Echocardiography, 7th ed. Lea and Febinger, Philadelphia.

17. FENELEY, M. P.; HICKIE, J. B. 1984. Validity of echocardiographic determination of left ventricular systolic wal thickening. Circulation 70: 226 .

18. FoRTUIN, N. J.; HoOD, W. P.; CRAIGE, E. 1972, Evaluation of left ventricular function by echocardiography. Circulation 46: 129-137.

19. HENRY, W. L.; A. DEMARIA; R. GRAMAK. 1980. Report of the American Society of Echocardiography committee on nomenclature and standards in two-dimensional echocardiography. Circulation 62: 212-217.

20. KIENLE, R. D.; W. P. THOMAS. 1995, Equine Echocardiography, in Nyland, T. G. and Mattoon, J. S. - Veterinary diagnostic ultrasound. $1^{\text {st }}$ Ed. W. B. Saunders Co.

21. KURAMOTO, K., A. SHIRAISHT; Y. NAKANISHI; M. KAU; Y. UENO; Y. UEDA. 1989. Application of echocardiography for assessing left ventricular function of Thoroughbred horses at a resting stage. Bull. Equine Res. Inst. 26: 23-30.

22. LESCURE, F; TAMSALI, Y. 1983. TM echocardiography in the horse. Point veterinaire, 15: 37-45.

23. LESCURE, F. AND Y. TAMZALI. 1984. Valeurs de reference en echocardiographie TM chez le cheval de sport. Rev. Med. Vet. 135: 405-418.

24. LiGHTOWLER, C.; M. MERCADO; J. GarCia LiÑEIRO; A. MOSCA. 1996. Ecocardiografia en el equino. Therios 25: 28-32.

25. Lightowler, C.; MERCADO, M.; Garcia LiÑEIRo, J.; PIDAL, G. 1998. The Echocardiographic Evaluation in the Horse. An Step by Step Approach. Proceedings CESMAS 98. Córdoba, España.

26. LightowleR, C.; G. PIDAL. 1999. Anatomía ecográfica del corazón equino. Un nuevo aporte para su conocimiento. Rev. Med. Vet, Bs. As. 80 (3): 233-239.

27. Lightowler, C.; Pidal, G.; García Liñeiro, J.; CATTANEo, M. 2000. Valor de referencia de la Separación Septal del Punto E en el caballo. Resúmenes Congreso Panamericano de Ciencias Veterinarias.

28. Lightowler, C.; Pidal, G.; García liñeiro, J.; CatTaneo, M. 1999. Evaluación de la función sistólica en el caballo. Parte 2. Velocidad de acortamiento circunferencial, \% de engrosamiento fraccional del tabique interventricular y $\%$ de engrosamiento fraccional de la pared posterior. Rev. Med. Vet. Bs. As. 80 (6): 515-519.

29. Lightowler, C.; Pidal, G.; García LÑ̃EIRO, J.; CaTtaneo, M. 2000. Evaluación de la función sistólica en el caballo. Parte 1. Fracción de acortamiento y Fracción de eyección. Arch. Med. Vet. XXXII (2).

30. Lightowler, C.; PIDAL, G.; Garcta Liñetro, J.; CatTaneO, M. 2000. Valores de referencia para los intervalos sistólicos en el caballo. Resúmenes Congreso Nacional, Chile.

31. LoNG, K. J.; J. D. Bonagura; P. G. G. DaRKE. 1992. Standardized imaging technique for guided $M$-mode and Doppler echocardiography in the horse. Equine Vet. J., 24: 226-235. 
32. LORD, P. F.; M. CROFT. 1990. Accuracy of formulae for calculating left ventricular volumes of the equine heart. Equine Vet. J., 9 (suppl): 53-56.

33. LONG, K. J. 1992. Two-dimensional and M-mode echocardiography. Equine Vet. Educ. 4: 303-310.

34. MARR, C. M. and V. B. REEF. 1995. Pathophysiology and diagnosis of cardiovascular disease, in Kobluk, C. N.; Ames, T. R. and. Geor, R. J. The Horse, Disease and clinical management. ${ }^{\text {st }}$. Ed. E. B. Saunders Co.

35. MARR, C. M. 1994. Equine echocardiography. Sound advice at the heart of the matter. Br. Vet. J. 150: 527-545.

36. O'CALLAGHAN, M. W. 1985. Comparison of echocardiographic and autopsy measurements of cardiac dimensions in the horse. Equine Vet. J., 17: 361-368.

37. O'CALLAGHAN, M. W. 1991. Echocardiography, in: Robinson, N. E. (ed). Current Therapy in Equine Medicine 2. Philadelphia: WB Saunders, pp. 151-159.

38. O'ROURKE, R. A.; P. HANRATH; W. N. HeNRY. 1984. Report of the joint International Society and Federation of Cardiology/World Health Organization task force on recommendations for standardization of measurements from $M$-mode echocardiograms. Circulation, 69: 854-857A.

39. Patteson, M. V.; C. Gibis; P. R. WotTon; P. J. CRIPPS. 1995. Echocardiographic measurements of cardiac dimensions and indices of cardiac function in normal adult Thoroughbred horse. Equine vet. J., Suppl. 19: 18-27.

40. PATteson, M. 1999. Two-dimensional and M-mode echocardiography. In Marr, C. (Ed.). Cardiology of the horse. 1st. Ed. W. B. Saunders Co. p. 102.

41. PAULl, K. S.; W. E. WINGField; J. J. BeRTONE. 1987. Echocardiographic changes with endurance training. In Gillespie, J. R.; Robinson, N. E. (eds): Equine Exercise Physiology 2. Davis. ICEEP Publications.

42. Pernoud, J.; Terdjman, M.; Kermaree, J. AND HafuenAUER, G. 1973. Myocardial contraction: study by ultrasonic echography (results in 200 normal patients) Nouv. Presse Med 2: 2393, 1973.

43. PIPERS, F. S.; R. HAMLIN. 1977. Echocardiography in the horse. J. Am. Vet. Medicine Ass. 170: 815-819.

44. PIPERS, F. S. 1978. Echocardiography in the horse. Proceedings of the $23^{\text {rd }}$ Annual Convention of the American Association of Equine Practitioners, pp. 173-176.

45. PIPERS, F. S., R. L. HAMLIN; V. REEF, 1979. Echocardiographic detection of cardiovascular lesions in the horse. J. Equine. Med. Surg. 3: 68-77.

46. QUINTONES, M. A.; W. H. GAASCH; J. K. ALEXANDER. 1976. Influence of acute changes in preload, afterload, contractile state and heart rate on ejection and isovolumetric indices of myocardial contractility in man. Circulation 53: 293-302.

47. REEF, V. B. 1990. Echocardiographic examination in the horse: the basics. Compendium Continuing Educ. Pract. Vet., 12: 1312-1319.

48. REEF, V. B. 1998. Equine Diagnostic Vltrasound. $1^{\text {st }}$ Ed., W. B. Saunders. p. 222.

49. SASAYANN, S.; FRANKLIN, D.; Ross, J.; KeMPER, W. S.; MCKown, D. 1976. Dynamic changes in left ventricular wall thicknes and their use in analyzing cardiac function in the conscious dog: a study based on a modified ultrasonic technique. Ann J. Cardiol 38: 870.

50. STEWERT, J. H.; R. L. ROSE; A. M. BARKO. 1984. Echocardiography in foals from birth to three months old. Equine Vet. J., 16: 332-341.

51. SAHN, D. J., A. DEMARIA; J. KISSLO; A.WEYMAN. 1978. The committee on $M$-mode standardization of the American Society of Echocardiography. Recommendations regarding quantitation in M-mode echocardiography: results of a survey of echocardiographic methods. Circulation 58: 1072-1083.

52. SCHIILER, N. B.; P.M. SHAN; M. CRAWFORD; A. DEMARIA; R. DevereuX; H. Feigenbaum; H. Gutgesell; N. ReICHEK; D. SAHN; I. SCHNITTGER; N. H. SILVERMAN; A. J. TAJIK. 1989. Recommendations for quantitation of the lefl ventricle by two dimensional echocardiography. American Society of Echocardiography Committee on Standards, subcommittee on Quantitation of Two Dimensional Echocardiograms. J. Am. Soc. Echocardiog. 2: 358-367.

53. SLATER, J. D.; M. E. HERRTAGE. 1995. Echocardiographyc measurements of cardiac dimensions in normal ponies and horses. Equine Vet. J. Suppl., 19: 28-32.

54. STADLER, P. REWEL, A.; DEEGEN, E. 1993. M-mode echocardiography in dressage and show-junping horses of class $S$ and in untrained horses, J. Vet. Med. A, 40: 292-306.

55. STAMM, R. B.; B. A. CARABELlO; D. L. MAYERS; R. P. MARTIN. 1982. Two-dimensional echocardiographic measurement of left ventricular ejection fraction: Prospective analysis of what constitutes an adecuate determination. Am. Heart. J. 104: 136-144.

56. StUarT, M.; A. SheikH; J. Gallastegui; G. T. Kondos; T. MANSON; W. LAM. 1982. Determination of left ventricular ejection fraction by visual estimation during real-time two-dimensional echocardiography. Am. Heart. J. 104: 603606.

57. Teichinolz, L. E.; T. Kreulen; M. V. HeRMan; R. GoRLIN. 1976. Problems in echocardiographic volume determinations: echocardiographic-angiographic correlations in the presence or abscence of asinergy. Am. J. Cardil. 37: 7-12.

58. TAYLOR, F. G. R.; M. H. MILLYER. 1997. Diagnostic techniques in equine medicine. $1^{\text {st }} \mathrm{Ed}$. W. B. Saunders Co.

59. ThOMAS, W. P., C. E. GaBer; G, J. JaCOBS; P. L. KaPLAN; C. W. LOMBARD; N. S. MOISE; B. L. MOSES. 1994. Recommendations for standards in two-dimensional echocardiography in dogs and cats. Vet. Radiol. Ultrasound, 35: 173-178.

60. Traill, T. A.; GIBSON, D. C. BROWN, D. J. 1978. Study of left ventricular wall thickness and dimension changes using echocardiography. Br. Heart J. 40: 162.

61. Voros, K.; HOLMES, J. R.; GIBBS, C. 1990. Anatomical validation of two-dimensional echocardiography in the horse. Equine Vet. J., 22: 392-397.

62. VoROS, K.; J. R. HOLMEs; C. GiBBs. 1990 . Left ventricular volume determination in the horse by two-dimensional echocardiography: an in vitro study. Equine Vet. J., 22: 398-402. 1083.

63. Voros, K.; J. R. Holmes; C. GibBs. 1991. Measurements of cardiac dimensions with two dimensional echocardiography in the living horse. Equine Vet. J., 1 (23): 461-463. 